\title{
STEPS to Enhance Physical Activity
}

National Cancer Institute

\section{Source}

National Cancer Institute. STEPS to Enhance Physical Activity. NCI Thesaurus. Code C156404.

A free-living physical activity intervention that uses pedometers, or other physical activity tracking, to reduce sitting time and other sedentary behaviors to improve fatigue, functional ability, muscle strength, physical activity, and quality of life, and promote positive health outcomes. 